\title{
A LOW-COST EFFECTIVE UWB LOCATION SYSTEM BASED ON FREQUENCY SYNTHESIS TECHNOLOGY
}

\author{
${ }^{1}$ Qilong Zhang and ${ }^{1,2}$ Fei Qin \\ ${ }^{1}$ Department of EEE, University of Chinese Academy of Sciences, Beijing, China \\ ${ }^{2}$ Key Laboratory of Wireless Sensor Network and Communication \\ SIMIT, Chinese Academy of Sciences, Shanghai, China
}

Received 2014-09-13; Revised 2014-11-18; Accepted 2014-12-10

\begin{abstract}
Wireless location system based on UWB signals has been widely utilized by wireless network recently, especially for the indoor scenarios where GPS signal has been heavily attenuated. However, due to the requirement of ultra wideband signals, existing location system usually faces problems including complexity, robust and cost, which limit the potential applications. In this study, we propose a new approach to achieve ultra wideband signal through frequency synthesis. The system will send series of narrow band signals and utilize signal processing algorithm in the receiver to obtain an effective ultra wideband signal but with significantly decreased complexity and cost. The simulation results validate the proposed approach, which may lead to the novel low cost RTLS system for wireless network.
\end{abstract}

Keywords: Indoor Location System, UWB, Frequency Synthesis, Location Estimation, TDOA

\section{INTRODUCTION}

Wireless indoor location system, or named as Real Time Location System (RTLS), usually refers to a wireless system with small Mobile Tags (referred as MT in this study) attached to the tracking object and a series of wireless Reference Stations (referred as RS in this study). MT and RS will exchange RF signals to estimate the relative location of MT. With the known positions of the pre-deployed Reference Station, the accurate location estimation of the Mobile Tag can be obtained. Unlike the GPS system, which cannot be utilized in the indoor scenarios (e.g., hospital, airport and warehouse) due to the heavy attenuation of signal and strong fading effect, the indoor location system can provide high accurate position data (around meters) in such environments. This has attracted great interests from industry, which pushes the research of indoor positioning system more than popular in recent years (Hui et al., 2007; Viani et al., 2011).

Several different approaches to implement UWB positioning system have been proposed in the past decade. Among which, the Ubisense product seems to have the best known accuracy around $15 \mathrm{~cm}$ through IRUWB (Impulse-Radio). The community thus investigated other UWB implementation approaches. The FMCW (Frequency Modulated Continuous Wave, also known as chirp) signal was introduced into the wireless location system by Nanotron, which achieves effective ultra wide bandwidth with significantly decreased complexity and cost (NT, 2014). But, a FMCW based system still requires costly wide intermediary frequency bandwidth architecture. Although numerous researchers demonstrated the advantage of FMCW signal for location system, the nonlinearity of its frequency variation rate, especially with wide bandwidth, may downgrade the quality of signal processing and limit the accuracy of position result (Huang, 2011).

To against the limitation of FMCW, step frequency synthesis technique has been proposed in this study to design a narrow IF bandwidth architecture. A frequency synthesis based UWB system will utilize series of narrow bandwidth signals to instead a single UWB signal but achieves similar accuracy, since the overall utilized Corresponding Author: Qilong Zhang, Department of EEE, University of Chinese Academy of Sciences, Beijing, China 
bandwidth is the same as single pulse UWB signal. In our architecture, each transmitter is an essentially narrow band signal and only requires narrow-IF system to transmit, which significantly decrease the complexity and power consumption of MT. The proposed novel architecture was designed with the aim of high accuracy, low complexity and more energy efficiency system for a reliable usage up to years. Other advantages include higher sensitivity by the narrower of bandwidth, which means the lower of noise floor leading to longer coverage.

To the best knowledge of us, Thales proposed a similar architecture named FH-UWB positioning system (Foy, 1976). But the Thales system is a Tag active system, which means the Mobile Tag must receive beacon signal from the Reference Station all the time to estimate its distance to each reference station. Therefore, although the system complexity has been decreased, the system was not essentially energy efficiency and can only be utilized in the emergency scenarios (exactly what Thales promoted to).

The organization of this study is as follows, chapter II will provide the system model of the step frequency synthesis location system. Chapter III will give numeric results to demonstrate the advantages. Finally, conclusion and future works will be discussed in Chapter IV.

\section{SYSTEM MODEL AND ESTIMATION ALORITHM}

\subsection{Signals under AWGN Channel and Ideal Sync}

In the proposed frequency synthesis based UWB system, a series of narrow band signals (named as a frame, while each signals named as a pulse) with step increasing carry frequency will be transmitted by the MT to cover an ultrawide frequency bandwidth. These narrow band signals will be received by the RS and be synthesized as a UWB signal to estimate the time differences. Finally, with the help of known locations of RS the estimated time differences could be utilized to calculate the unknown location of MT.

Suppose the pulse cycle in each Frame $T_{r}$ is, the width of pulse is $\tau$, the base frequency is $f 0$, the step of frequency increscent is $\Delta f$, the pulse number is $N$ and the speed of signal is $c$. Then, the signals inside a frame can be described as follows:

$$
x(t)=\sum_{i=0}^{N-1} r e c t\left(\frac{t-i T_{r}-\frac{\tau}{2}}{\tau}\right) \exp \left(-j 2 \pi\left(f_{0}+i \Delta f\right) t\right)
$$

The RS with distance $R$, will have the signal with a simply time delay of and an AWGN noise item. If we assume the receiver device has already achieved perfect time and frequency synchronization (this is an idea assumption in implementation, we will relax this constraint with TDOA architecture later in this section). The mixture of transmit signal and received signal will have the following output Equation 2:

$$
y(t)=\sum_{i-0}^{N-1} \exp \left(-j 2 \pi f_{0} \frac{R}{c}\right) \exp \left(-j 2 \pi i \Delta f_{0} \frac{R}{c}\right)+w(n)
$$

In which, the second exponential item contains the frequency synthesis information between pulses, i.e., resolution was related to the overall bandwidth $B=N \Delta f$.

$$
S(k)=\sum_{i=0}^{N-1} \exp \left(-j 2 \pi \frac{2 R i \Delta f}{C}\right) \exp \left(j 2 \pi \frac{i k}{N}\right)
$$

The distance information was expressed with the phase items among received pulse in a frame. Therefore, by converting the signal with IFFT processing shown in Equation (3), the distance estimation with high resolution item can be simply calculated with the location of peak in the spectrum (i.e., the maximized value $S(k)$ can only be obtained with $\frac{2 R \Delta f}{c}=\frac{k}{N}$ ).

Now with the estimated distance and at least 3 known locations of RSs, the unknown location of MT can be uniquely calculated through the cross point of three cycles. Since the technology of this process has been well developed in navigation and GPS system (Foy, 1976) we will omit the discussion in this study.

It should be noted that, each wireless pulse is essentially a narrow band signal, which significantly decrease the complexity, consumption and cost of wireless transceiver. The whole system, in turn, will rely on the switching of multiple carrier frequencies to obtain the effective UWB signal. This architecture is very mature in most existed short range wireless system in the form of multi-channel capability, including IEEE 802.11, IEEE 802.15.4 and Bluetooth. For example, IEEE 802.15.4 signal covers $2 \mathrm{MHz}(5 \mathrm{MHz}$ with guard band) in each channel, but there are 16 channels supported in IEEE 802.15.4 system, which spread from $2.405 \mathrm{GHz}$, to $2.480 \mathrm{GHz}$, cover $80 \mathrm{MHz}$ in total. Through the employment of proposed approach, an IEEE 802.15.4 system could obtain an effective 
location accuracy of $80 \mathrm{MHz}$ (the system will need slightly modification in-deed), instead of the low accuracy of $2 \mathrm{MHz}$ narrow band system.

The other advantage of proposed architecture is the higher sensitivity bring by the narrow band signal. The sensitivity decides the coverage of a RTLS system following Shannon theory. Since the proposed frequency synthesis system was essentially a multiple narrow band system, the effective noise bandwidth of each pulse can be approximated by, while the noise bandwidth of IRUWB and FMCW based UWB was decided by. As a result, the noise floor of proposed architecture could be much lower, which contributed to much better sensitivity and coverage.

However, to obtain the advantages discussed above, the system has to pay the expense of time cost. In detail, each estimation will require the transmission of whole frame which lasts much longer than single pulse. Considering that RTLS system usually requires the update rate with only several $\mathrm{Hz}$ or even lower and the transmission of whole frame only lasts tens of micro second, i.e. corresponding to hundreds $\mathrm{Hz}$ of update rate. Therefore, it was wondering that this drawback can be afforded in modern RTLS system.

\subsection{TDOA Architecture}

It is usually more than difficult to implement an ideal synchronization between transmitter and receiver due to the wireless and mobile nature of MT in the real life implementation. As a result, the received signal cannot be simply mixed with the exactly recovered transmit signal as we shown in section 2.1. Some researchers (Harmer et al., 2008), rely on the two-way time of flight method to solve this synchronization problem. However, this approach requires the MT keeps receiving the incoming signals and returns an invoked copy. The signal processing will be carried out in the transmitter side, where the ideal local frequency is generated or stored. This approach significantly increased both the power consumption and the hardware complexity of MT, which is energy constraint in nature yet is not suitable for this architecture (Harmer et al., 2008).

As shown in Fig. 1, the other choice of low cost location systems is the Time Difference of Arrival (TDOA), where the system detects the time difference of the same signal transmitted from MT to different RSs. The TDOA architecture waives the requirements of time synchronization between MT and RS and only requires the synchronization among RSs. This can be simply implemented through RF cable backbone or radio over fiber technology (Huang, 2011), which will all feed all received signals from RSs into a central sever to mix with each other. Consequently, the Equation (2) should be rewritten as following (also omit the low resolution item) to estimate the distance difference between RS1 and RS2 Equation 4:

$$
y(t)=\sum_{i=0}^{N-1} \exp \left(-j 2 \pi i \Delta f \frac{R 1-R 2-r 1^{\prime}-r 2^{\prime}}{c}\right)+w(n)
$$

where, is the distance between MT and RS1, is the distance between MT and RS2, $r 1^{\prime} r 2^{\prime}$ are the effective transmission delays from RS1 and RS2 to the central server (can be measured with offline pattern).

However, the TDOA architecture will change the location estimation method as well, which will try to obtain the cross point of two hyperbolas. Thus, the location can be estimated by solving the following Equation 5:

$$
\left\{\begin{aligned}
& \Delta R_{1,2}=\left(\begin{array}{l}
\sqrt{\left(x_{t}-x_{1}\right)+\left(y_{t}-y_{1}\right)^{2}}- \\
\sqrt{\left(x_{t}-x_{2}\right)+\left(y_{t}-y_{2}\right)^{2}}
\end{array}\right) \\
& \Delta R_{1,3}=\left(\begin{array}{l}
\sqrt{\left(x_{t}-x_{1}\right)+\left(y_{t}-y_{1}\right)^{2}}- \\
\sqrt{\left(x_{t}-x_{3}\right)+\left(y_{t}-y_{3}\right)^{2}}
\end{array}\right)
\end{aligned}\right.
$$

where, $\Delta R_{1,2}$ and $\Delta R_{1,3}$ are the distance estimation out-puts, $\left[x_{t}, y_{t}\right]$ is the location of MT, $\left[x_{1}, y_{1}\right]\left[x_{2}, y_{2}\right]\left[x_{3}, y_{3}\right]$ are the known locations of three RSs.

Easy to note that, this architecture can significantly decreases the power consumption and complexity of MT (by shifting to the RS side). Considering that RS will usually be permanently installed and can easily obtain power, the additional synchronization and calculation cost is affordable.

\section{EXPERIMENT VALIDATION}

In this section, series of simulation based experiments will be implemented to demonstrate the validity and efficiency of proposed frequency synthesis based location system. The simulation was based on the IEEE 802.15.4 system with necessary modification of proposed architecture. In detail, each pulse occupies $2 \mathrm{MHz}$ bandwidth and between pulses is set to $2 \mathrm{MHz}$ as well. Each frame will transmit 40 pulses covers $80 \mathrm{MHz}$ bandwidth in total.

Figure 3 provides the leading two pulses of a frame, in both time domain and frequency domain. Easy to notice that pulse 1 and pulse 2 have the same bandwidth, only the carrier frequency was shifted with $\Delta f$. 


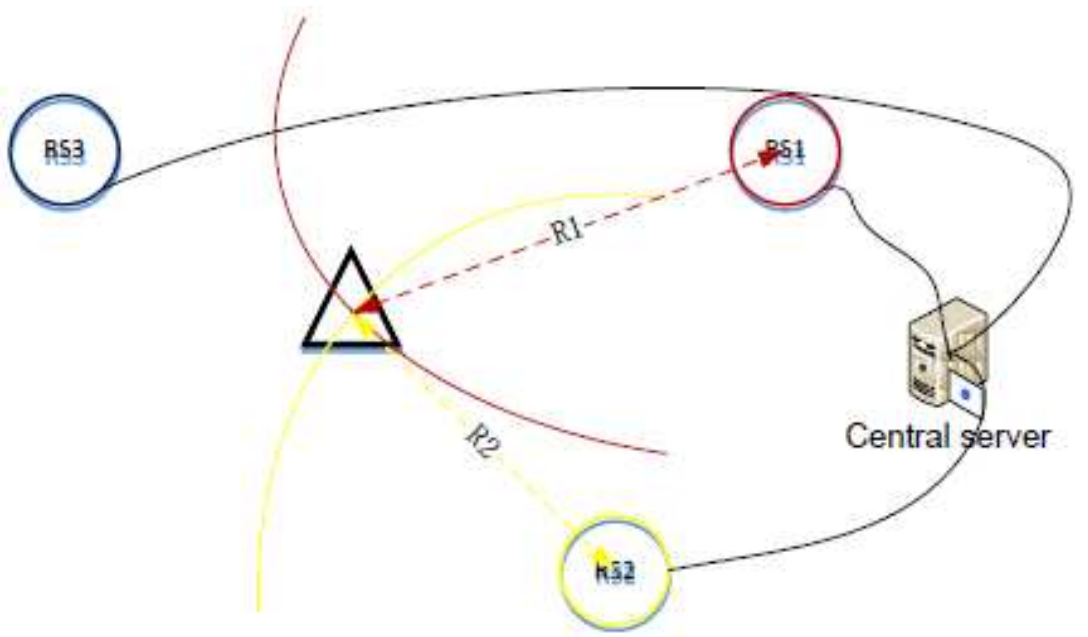

Fig. 1. Example of proposed wireless location system

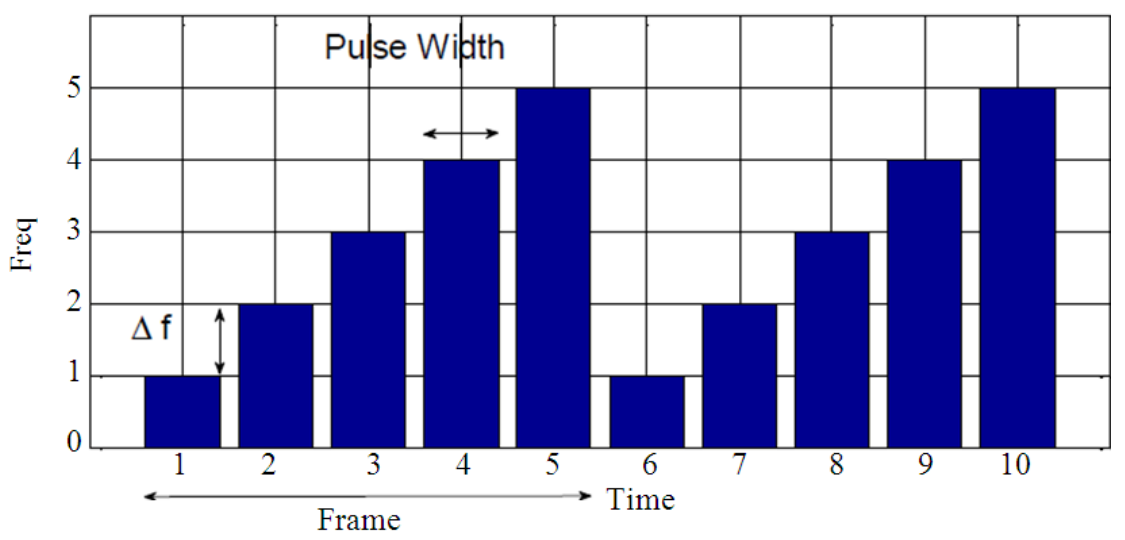

Fig. 2. Scheme of step frequence synthesis

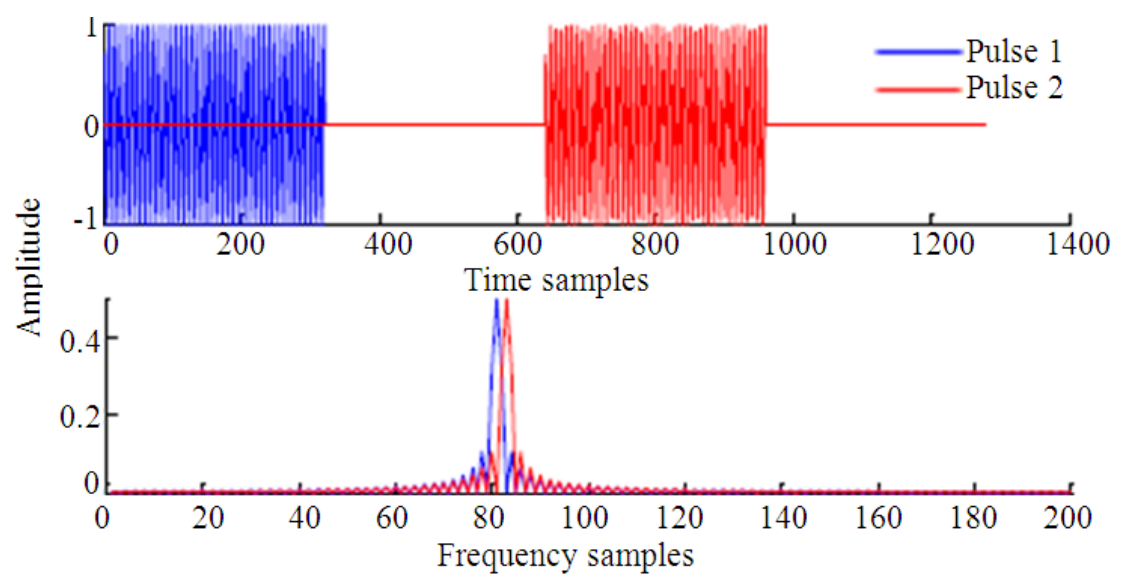

Fig. 3. Waveform of pulses 
If all the 40 pulses have been plotted, the frequency bandwidth will cover the overall $80 \mathrm{MHz}$ as shown in Fig. 4, which demonstrates the basic idea of proposed effective UWB system.

Figure 5 provides the scheme of processing in the receiver side. Due to the different distance between MT and two RSs, the signals transmitted from the MT will arrive two RSs with a small time difference. This time difference can be utilized to calculate the distance difference between two RSs, which is usually termed as low resolution result. The accuracy of low resolution item was contributed by three factors: Bandwidth of signal, ADC rate and accuracy of time synchronization. The bad news is that the final system accuracy was decided by the worst factor. For example, even if idea time synchronization has been assumed, the accuracy of distance estimation with the low resolution item will be decided by the signal bandwidth, i.e. $2 \mathrm{MHz}$ in this simulation which contributed to tens of meters estimation error. It worth noted that the low resolution result will not related to the carrier frequency, therefore, although 40 pulses will be transmitted, the sys-tem performance will be the same as a single pulse.

However, as we discussed in section 2, the second exponential item of mixed signals within a frame can be utilized to estimate a high accuracy, i.e. effective UWB signal. The basic scheme has been shown with the black line in Fig. 5. Due to the different carrier frequency, the output amplitude of each pulse will be different, the IFFT process of which will bring us the high resolution estimation result. Figure 6 provides the 3D result of the IFFT process: The X-axis is the low resolution result, while the IFFT process will generate the high resolution result in y-axis. The final distance estimation will be decided by both the bandwidth of single pulse and pulse numbers, which is 3.75 meters (effective to 80MHz UWB signal).

By manually setting the distance between two RSs, the results shown in Fig. 7 demonstrate the relationship of real distance and estimated distance. Easy to notice that the minimal resolution is 3.75 meters when $\mathrm{B}=80 \mathrm{MHz}$, which demonstrates the frequency synthesis architecture can obtain the effective UWB signal's performance. It is very straightforward to further increase the distance estimation accuracy with more pulse numbers (i.e. cover wider bandwidth, say $400 \mathrm{MHz}$ in Fig. 7).

Through position estimation algorithm, e.g., TaylorSeries Estimation algorithm (Foy, 1976), the distance estimation result in TDOA architecture can contribute even higher location estimation results. As shown in Fig. 8, the output results will be compared with generated location, then the residue error will be recorded: The 3.75 meters distance resolution will result in a location estimation error of no more than 1 meter.

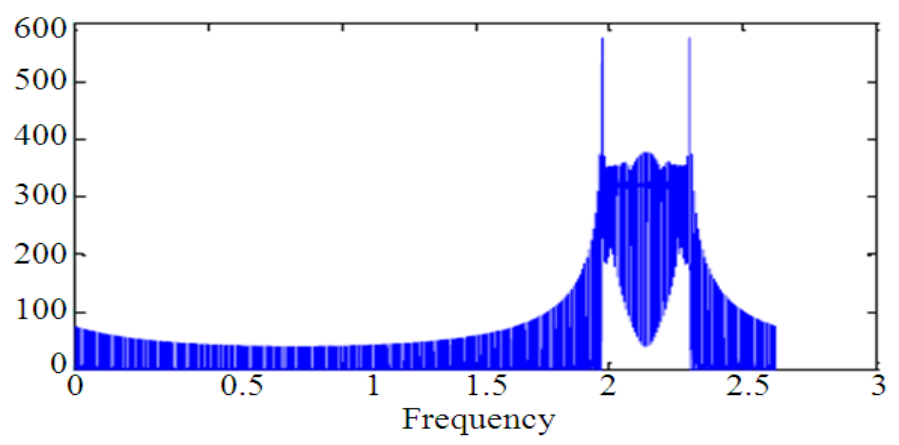

Fig. 4. Frequency domain of the whole frame signals

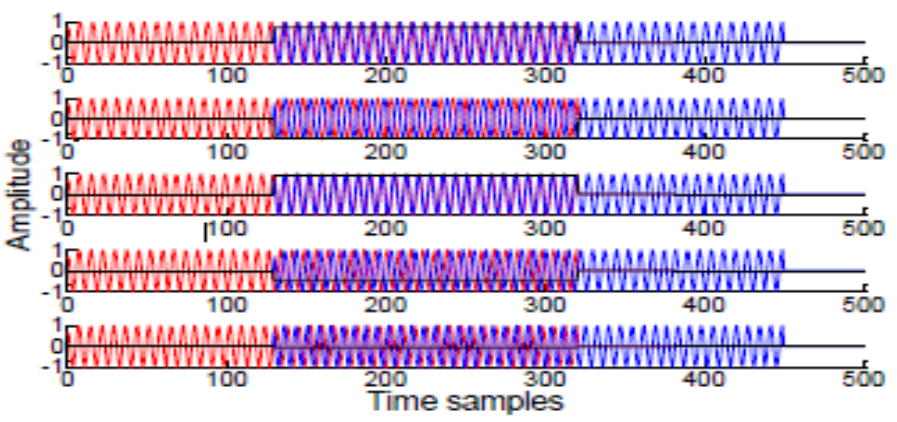

Fig. 5. Scheme of signal mixture 


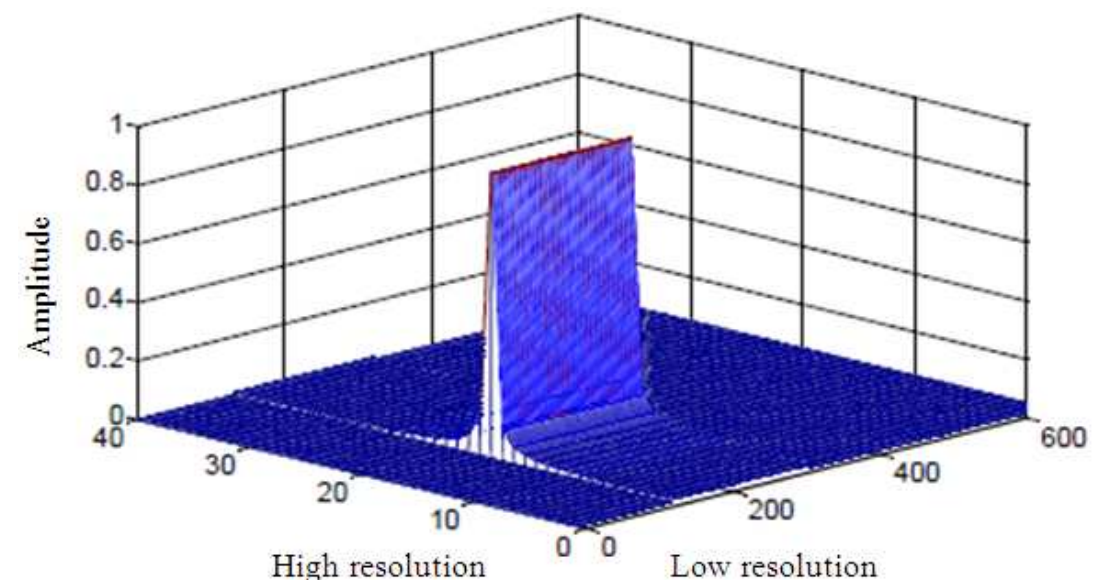

Fig. 6. Scheme of frequency synthesis

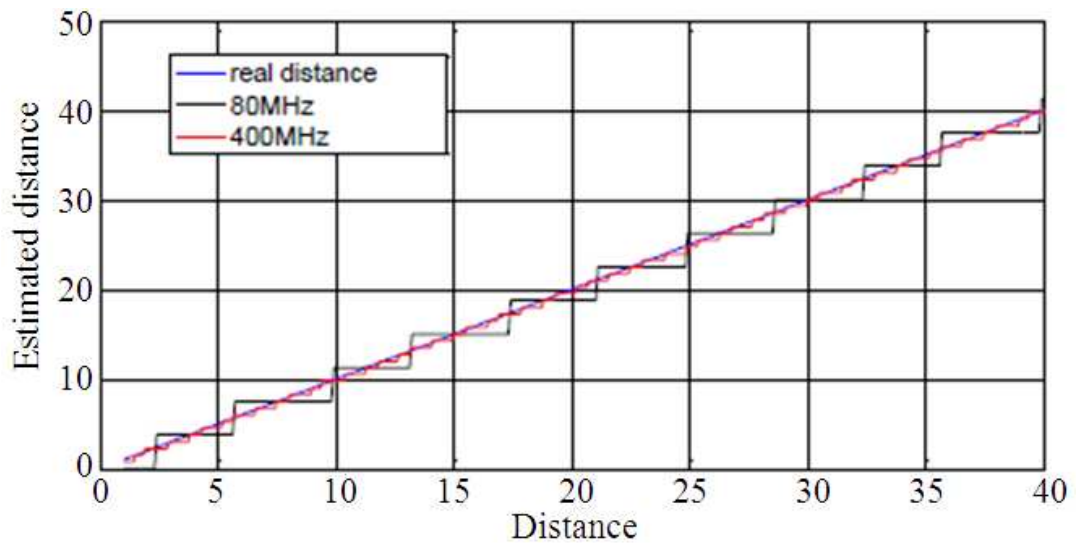

Fig. 7. Distance estimation reuslts

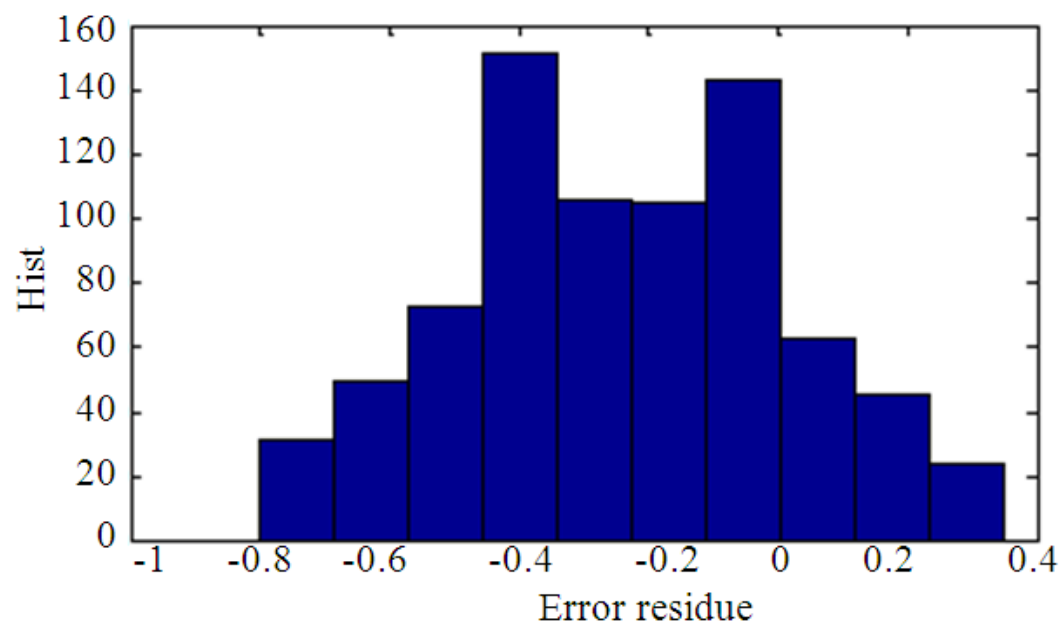

Fig. 8. Estimation accuracy 


\section{CONCLUSION}

In this study, we show a novel architecture of frequency synthesis based UWB location system through theoretical analyses and simulation result. The experiment demonstrates the great potential of the proposed architecture with significantly increased the location accuracy. However it is easy to understand that the narrow band-width will suffers from the strong indoor multi-path fading effects, which prevents its ability to achieve high ac-curacy. In the future work, we will investigate how to against the effect of multi-path fading, as well as try to deploy proposed system into Software Defined Radio based system to give a comprehensive performance re-view and modify proposed algorithm with feedback from the experiments.

\section{ADDITIONAL INFORMATION}

\subsection{Funding Information}

This study was supported by the Nature Science Foundation of China under the grant No. 61401426 and by the Funding Scheme of Key Laboratory of Wireless Sensor Network and Communication, Chinese Academy of Sciences (Grant No. 2013004) and also by the President Fund of University of Chinese Academy of Sciences (Grant No. Y35102JN00).

\subsection{Author's Contributions}

Mr. Qilong Zhang: Carry out the whole theory analyses, experiment design and paper writing.

Dr. Fei Qin: Proposed the original idea of this paper and help to write the paper.

\subsection{Ethics}

To the best of out knowldege, there is no similar work existed and we are the first to propose such a low cost, low power consumption and low IF bandwidth architecture for the wireless indoor locating system.

\section{REFERENCES}

Foy, W.H., 1976. Position-Location Solutions by TaylorSeries Estimation. IEEE Trans. Aerospace Elect. Syst., 12: 187-194. DOI: 10.1109/TAES.1976.308294

Harmer, D., M. Russell, E. Frazer and T. Bauge, 2008. EUROPCOM: Emergency ultrawideband radio for positioning and communications. Proceedings of the IEEE International Conference on UltraWideband, Sept. 10-12, IEEE Xplore Press, Hannover, 85-88. DOI: 10.1109/ICUWB.2008.4653422

Huang, Y, 2011. A study of RF-over-fibre based active RFID indoor location system. PhD Thesis, University College London.

Hui, L., H. Darabi, P. Banerjee and L. Jing, 2007. Survey of wireless indoor positioning techniques and systems. Proceedings of the IEEE Transactions Systems, Man and Cybernetics, Part C: Applications and Reviews, System Man Cybernetics, (SMC' 07), IEEE Xplore Press, pp: 1067-1080. DOI: 10.1109/TSMCC.2007.905750

NT, Nanotron, 2014. Chirp spread spectrum. Nanotron's Technology GmbH.

Viani, F., P. Rocca, G. Oliveri, D. Trinchero and A. Massa, 2011. Localization, tracking and imaging of targets in wireless sensor networks: An invited review. Radio Sci. DOI: 10.1029/2010RS004561 